\title{
Understanding Emerging Design Practices for Avatar Systems in the Commercial Social VR Ecology
}

\author{
Anya Kolesnichenko \\ Santa Cruz, CA, USA \\ UC Santa Cruz \\ akolesni@ucsc.edu
}

\author{
Joshua McVeigh-Schultz \\ Santa Cruz, CA, USA \\ UC Santa Cruz \\ jmcveigh@ucsc.edu
}

\author{
Katherine Isbister \\ Santa Cruz, CA, USA \\ UC Santa Cruz \\ katherine.isbister@ucsc.edu
}

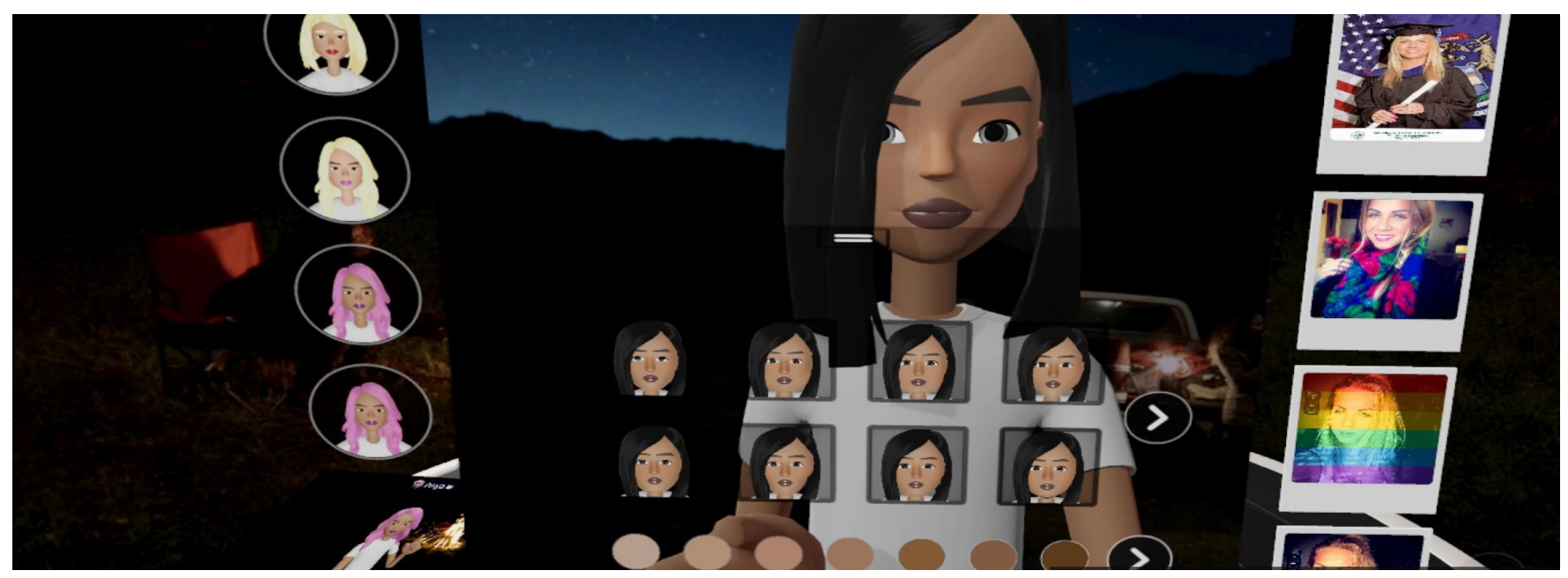

Figure 1: Avatar Representation Affordances in Facebook Spaces

\section{ABSTRACT}

In the emerging ecology of commercial social VR, avatars that serve to represent individuals within these multi-user virtual worlds are at the heart of the embodied social experience. Current industry approaches to avatars in social VR applications vary widely, and the (sometimes tacit) design knowledge acquired by those who created these platforms has much to offer research in HCI. In this paper, we describe current design practices, and reflect on the design approaches that characterize avatars and avatar systems in this emerging commercial sector. To investigate design approaches to avatar systems and their impact on communication and interaction with people within this medium, we interviewed industry experts associated with a range of platforms including Rec Room, AltspaceVR, High Fidelity, VRChat, Anyland, and Mozilla Hubs. In analyzing the ways that design choices shape embodied experience, we map design approaches to avatar systems in this evolving landscape and make preliminary claims about the impact of these varying design approaches.

Permission to make digital or hard copies of all or part of this work for personal or classroom use is granted without fee provided that copies are not made or distributed for profit or commercial advantage and that copies bear this notice and the full citation on the first page. Copyrights for components of this work owned by others than ACM must be honored. Abstracting with credit is permitted. To copy otherwise, or republish, to post on servers or to redistribute to lists, requires prior specific permission and/or a fee. Request permissions from Permissions@acm.org.

DIS '19, June 23-28, 2019, San Diego, CA, USA

(C) 2019 Association for Computing Machinery.

ACM ISBN 978-1-4503-5850-7/19/06\$15.00

https://doi.org/10.1145/3322276.3322352

\section{Author Keywords}

Avatar design; avatar system; social VR; virtual identity; avatar representation; virtual multi-user worlds;

\section{CCS Concepts}

- Human-centered computing Virtual reality • Humancentered computing Empirical studies in collaborative and social computing • Human-centered computing $\sim \mathrm{HCI}$ design and evaluation methods • Human-centered computing Collaborative interaction • Human-centered computing Open source software

\section{INTRODUCTION}

As virtual reality (VR) headsets have become more commercially affordable, a growing number of social VR platforms have continued to adapt these technologies for wider public use. As the embodied interfaces and proxies through which users enter VR, avatars represent a core aspect that shapes the experience of those in social VR [40]. For instance, avatar features serve an important role in how people communicate both verbal and non-verbal information to others in VR [30],[32],[34],[36].

Previous research on avatars in VR tends to focus on the impact of specific avatar differences in laboratory conditions rather than considering the role that avatars play in broader social VR ecologies. Likewise, while these sorts of ecological perspectives inform studies of avatars in 2D screen-based environments (such as Second Life), the medium of VR heightens the stakes of avatar embodiment in several key ways, including navigation modalities, avatar 
aesthetics, social mechanics, and personal space management.

What kinds of embodied experiences do commercial social VR platforms support? What are the challenges, trade-offs, constraints and stakes involved in avatar design practices for social VR? What are the nuances and unique opportunities that this medium holds for the representation of our virtual selves? Due to the shift towards communication technologies and cross-platform applications, the variety of uses in these technologies sometimes overlap and are often being used for similar purposes. This creates a need for the research community to understand how the emerging design practices for embodied social VR experiences support interpersonal communication and spatial interaction. What are the commonalities and differences between those practices?

To address these questions, we drew upon expert interviews to map a slice of the emerging ecology of avatars in social VR. We interviewed designers, developers and other experts from a range of social VR applications including: AltspaceVR, Mozilla Hubs, VRChat, Facebook Spaces, Anyland, and High Fidelity. While these interviews concentrated on creators, most of their perceptions of associated user experiences were based on internal user data and empirical observations they used to support their arguments, which we clarified during interviews. By unpacking the various ways that these applications handle avatars, we also make the claim that research on avatars in VR should focus on avatar systems as the defining framework for how people experience avatars in social VR.

This research foregrounds a timely opportunity for researches to understand the relationship between design decisions, platform specifics, social contexts, and emerging design practices pertinent to embodied user experience in social VR. We use the term avatar system to convey the importance of multiple factors and dimensions that shape the quality of embodied experience within social VR design topology. These dimensions include embodied locomotion, avatar aesthetics, personal space, social mechanics, and avatar's relation to virtual identity. Differences in avatar mechanics and affordances that may seem minor at first glance can have a significant impact on social dynamics that develop in social VR. Our understanding of avatar systems was drawn from both the prior scholarly work on avatars, and new emerging design patterns we found in the commercial social VR medium.

\section{BACKGROUND}

The study of "virtual humans" [2], also known as avatars, is informed by a long history of research that has significantly progressed over the past two decades. The concept of an avatar and its multi-faceted dimensions has also evolved, with the emergence of immersive communication technologies. Because people are embodying themselves in social VR in multivalent ways, we decided to re-visit the question about what an avatar is, and how research on avatars and their counterparts can be most meaningfully handled in multi-user virtual worlds.

The first appearance of the concept of an "avatar" can be traced back to 1970 in works of fiction. The term "avatar" was first introduced in 1984 in online multiuser dungeons or so-called $M U D s^{1}$ [1]. Neal Stephenson, author of the science fiction novel Snow Crash (published in 1992), is also known for being the first person to apply the term "avatar" in a completely new sense, adopting the label for the digital representation of a person in virtual environments [35]. According to the Encyclopedia of Human-Computer Interaction (2004) and other sources, the origin of the term "avatar" is as follows:

... "Avatar derives from the Sanskrit word avatarah, meaning "descent", and refers to the incarnation - the descent into this world - of a Hindu god. A Hindu deity embodied it's spiritual being when interacting with humans by appearing in either human or animal form." [1],[26].

Avatars play an important role in shaping how social life emerges and develops in multi-user virtual worlds, and how users communicate with one another [9],[15]. In the social VR medium, avatars are embodied constructs that inhabit virtual worlds [30]. According to a recent study by Waltemate et al., avatars were found as "users' embodied interfaces to and their proxy" within immersive virtual environments [40]. Bosch-Sijtsema et al. believed that avatars served as a user's "protective shield" that ensures their comfort and safety in social contexts within multi-user virtual worlds [4]. Jacquelyn Ford Morie argued that an avatar as our projected self is what we let others see in a virtual world [24]. It is true that other users in virtual worlds of social VR get to know us through our avatars. They learn about us through our avatar's look, movements, our voice, communication patterns, and through how we determine our interests and affiliation to social groups. Morie and Garau et. al have claimed that avatars in social VR systems do not yet have as wide a range of expressivity as humans have in the physical world [12],[24]. However, in today's commercial social VR medium, this has improved to some degree. Designers have managed to balance the degree of an avatar's expressivity, and the constraints related to cross-platform compatibility aspects between the web and VR, including software and hardware specifics.

Research on avatars has included studies on avatar creation, its personalization affordances, and their impact on body ownership, presence, and dominance compared to generic counterparts [5],[7],[38]. Studies on avatars in collaborative virtual environments have found that extended possibilities to express oneself reinforce creative activities and social interaction within virtual environments [6],[8],[13],[27],[31],[33]. Research on self-projection and

\footnotetext{
${ }^{1}$ MUDs - role-playing environments.
} 
the sense of proximity contributed to the avatars' potential to function as a self-representation of a virtual community member [13]. Research on userss identification with avatars argued for their facilitation of cultivating intrinsic motivation within and beyond virtual spaces [3].

Research on factors that define avatar aesthetics has also included a long history of experiments that relate to visual and behavioral dimensions of avatars [2],[11],[23],[28],[39]. While Bailenson, Beall, and Fox explore how the manipulation of appearance and behavior in avatars can be exploited for different purposes [1],[2],[10], Garau investigated the fidelity of avatar appearance, with special reference to behavioral realism and eye gaze [12],[32],[39]. The study of neurological traces from interacting with lookalike avatars confirmed participants' tolerance in the processing of self-faces even when they are distorted [14].

A myriad of studies on avatars in multiplayer video games have empirically demonstrated that player avatars that represent their real-self reinforce identification [16],[19],[37]. Some researchers, however, found that players could sacrifice avatar-player convergence (harming identification) for strategic reasons in competitive play [3].

Our own preliminary research found commercial social VR to be part of a diverse and evolving media landscape [21],[22],[25]. In this prior research we mapped a slice of the social VR ecology in terms of aesthetics, theme, functionality, interaction mechanics, and emergent social norms [21],[22]. Aside from this preliminary work on commercial VR platforms, less has been done to address the specifics of design approaches to avatar systems in social VR applications, a factor that this research foregrounds.

\section{METHODS}

To investigate design practices for avatar systems in the commercial social VR medium, we conducted interviews with experts in design and development affiliated with six popular social VR applications: Rec Room, High Fidelity, AltspaceVR, VRChat, Facebook Spaces, and Anyland. This study was designed based on similar design research methods that draw upon expert interviews to develop a framework [17],[18],[22]. This section outlines research participants' demographics and their backgrounds, followed by a description of our study design, procedure, data collection, and analysis methods.

\section{Participants}

We interviewed 11 experts who represent a wide range of roles and backgrounds in the social VR industry, including designers, engineers, and developers. Our criteria for selecting the participants were that they had participated in or led the design and development process in commercial social VR.

\footnotetext{
${ }^{2}$ Manyland official web site - http://manyland.com/
}

\section{Demographics}

Interview experts included 6 women, 4 men, one identified as "cyborg", and one who declined to respond. They came from various locations within North America, Canada and Europe (one participant stated their national origin as “artificial boundaries shouldn't exist").

\section{Roles and Backgrounds}

Ten out of 11 participants granted their permission to reveal their real names in public facing venues. Below is a breakdown of the roles and backgrounds of interviewees:

- Avatar Designer, Game Artist from Morph3D, also a Content Creator for VRChat; worked in VR since December 2017.

- Philipp Lenssen, Developer and Co-founder of Anyland; Philipp had been releasing updates to Anyland daily since its first launch on Steam in October 2016; he has a sibling 2D project, called Manyland ${ }^{2}$.

- Corey Nolan, Gameplay Programmer at Rec Room; first job in VR industry.

- Adam Dormier, Community Support Coordinator at Rec Room; Adam was an avid player of Rec Room for about 1.5 years prior to being recruited by Against Gravity ${ }^{3}$ in 2017.

- Tamara Hughes, Community Support Coordinator at Rec Room; Tamara was an avid player of Rec Room for over a year before she joined the team at Rec Room.

- Alexia Mandeville, UX Designer on a platform team at High Fidelity VR; Alexia worked in the VR industry for 5 years, previously did game and haptic design for VR/AR technologies.

- Greg Fodor, Software Engineering Manager at Mozilla Hubs; Greg was one of the co-founders of AltspaceVR, prior to its' official affiliation with Microsoft in October 2017.

- James (Jim) Conrad, Mixed Reality Designer at Mozilla Hubs; Prior to joining the team at Mozilla (September 2017), Jim was a Senior Designer at Crystal Dynamics on a Tomb Raider (2013 reboot) project, and a Designer at AltspaceVR.

- Miriam Avery, Director of Strategic Foresight at Mozilla Hubs; Forecast Strategist and Qualitative Researcher.

- Evan Sforza, Mixed Reality Designer at AltspaceVR (Microsoft) for 2 years; In the past, Evan was a Multiplayer Designer at 343 Industries on Halo 5: Guardians project.

- Ishita Kapur, Senior Product Manager at AltspaceVR (Microsoft); Ishita has been with Microsoft for 7.5 years, and worked in VR, AR and Mixed Reality for 2.5 years.

\footnotetext{
${ }^{3}$ Against Gravity are makers of Rec Room, official web site https://www.againstgrav.com/
} 


\section{Recruitment}

Interview participants were recruited through our personal and professional networks; social media platforms, such as Twitter, Facebook, including both public and private virtual reality events, such as Silicon Valley Virtual Reality (SVVR) meetups ${ }^{4}$, and Museum of Other Realities (MOR) ${ }^{5}$ meetups. For VRChat and Facebook Spaces we were unable to find creators who would participate in our research directly, possibly due to discomfort with sharing proprietary details. Instead, for Facebook Spaces we drew insights from public articles and design blogs written by key product designers. For VRChat we interviewed a $3^{\text {rd }}$ party content creator.

\section{Study Design}

The preliminary findings drawn from our previous landscape research [21] informed the design of questions for the expert interviews. This section introduces interview topics pertinent to avatar systems, and describes the format of conducted interviews.

\section{Interview Topics}

Interview topics related to avatars in social VR were broken down into the following subtopics: (1) Embodied Locomotion (i.e. What is the design approach to locomotion in [APPLICATION]? How do users get around and how do they explore?); (2) Personal Space (i.e. What is the design approach to personal space of avatars in social contexts? How [APPLICATION] addresses harassment? Have experts fielded any complaints from their users?); (3) General Avatar Design Approach - (i.e. What is the avatar design process? What are the design goals? How does avatar design relate to the kinds of social interactions that [APPLICATION] is designed to support?); (4) Avatar Appearance and Customization - (i.e. How did experts approach avatar features (representation)? Does the application invite users to customize or select avatars in a particular way?); (5) Perception of Others - (i.e. How do experts think avatars influence the way users react to others in social contexts?); (6) Avatars and Identity - (i.e. When experts were designing the system for avatars, what kind of identities did they have in mind for your users?); (7) Avatar Mechanics - (i.e. What kinds of actions can avatars do?); (8) Social Mechanics (i.e. How experts designed the friending system (if it exists) that allows users connect with others in [APPLICATION]?).

\section{Interview Format}

All interviews were conducted in the social VR application that the interviewee was affiliated with. We identified the following key benefits in conducting expert interviews in VR setting: (1) Instant Demonstration of Referenced Design Features - interview topics were instantly available for participants to showcase them in real time; (2) Synchronized Visual and Audio Data Collection - we were able to video and audio capture all interactions that occurred during the inworld interviews.

\footnotetext{
${ }^{4}$ Silicon Valley Virtual Reality meetup group web site https://www.meetup.com/SiliconValleyVirtualReality/
}

\section{Procedure}

The duration of expert interviews ranged from 1.5 to 2 hours, depending on the availability of interview participants. Most interviews were individual, and some were group interviews with up to 3 participants being present in the same virtual space [Figure 2]. We found people with different expertise could help fill in different parts of the puzzle associated with a certain interview topic.

\section{Logistics}

The interviews were conducted by two researchers - one used their desktop computer while the other one interacted with the participant within the virtual world in real time wearing a VR headset (either Oculus Rift, or HTC Vive). Researchers took notes and coordinated timing using their desktop computer during the interview.

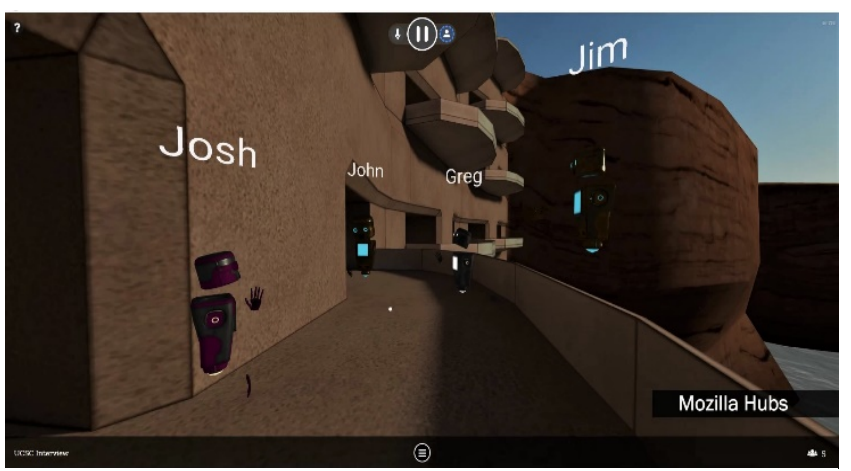

Figure 2: Example of Group Interview in Mozilla Hubs.

\section{Analysis Methods}

We applied a hybrid of three methods common for expert interviews to study the industry design practices: (1) Openended interview questions to gauge the landscape of design approaches; (2) Qualitative analysis of answers and observation notes [17],[18]; (3) Analysis of recorded video demonstrations related to avatars and embodied experience.

Our design research team brought together backgrounds in game design, psychology, social science, computational media, as well as experience in designing prototypes for social VR. Each of us reviewed the video recordings of interviews and made the detailed notes of our own. We used semi-automated transcription tools linked to video. All notes were documented in spreadsheets that were shared among co-investigators.

We worked together to categorize responses. We applied cues from Saldana's approach to qualitative analysis of interviews [29]. For each interview topic, we shortlisted the most important quotes. We then collectively clustered the quotes, or some parts of them into the following design practice categories for: (1) Embodied Locomotion (teleportation, flying, jumping, walking); (2) Avatar Aesthetics (visual and behavioral fidelity, avatar selection, appearances, customization); (3) Personal Space (avatar

\footnotetext{
${ }^{5}$ Museum of Other Realities (MOR) official web site - http://th-er.com/
} 
personal bubbles); (4) Social Mechanics (friending, emoting, muting, blocking, etc.); (5) Avatar's Relation to Virtual Identity (such as personalization affordances of avatars). We compiled the generated notes and looked for overarching themes, commonalities, constrains, trade-offs, and the stakes involved.

\section{FINDINGS}

Our findings identified several novel affordances of embodiment for avatars in social VR, including the following: (1) bringing real-world identities into users' avatars through image recognition software (i.e. a photogenerated personal avatar in Facebook Spaces); (2) creating a party with other users to perform an activity together through the avatar's fist bump gesture (i.e. group travel in Rec Room); (3) uploading media files into the virtual space to support collaboration (i.e. import media in $V R$ in MozillaHubs); (4) making platform creation tool sets that allow users to create their own worlds and upload custom avatars (i.e. platform creation tools in High Fidelity); (5) having cross-platform compatibility of built-in affordances of avatar mechanics (i.e. cross-platform support in AltspaceVR); (6) developing in-world creations and avatars without taking a headset off (i.e. in-world creation tools in Anyland); (7) creating custom avatars and adding custom non-verbal behavioral functionalities to it (i.e. import custom avatar in VRChat). Table 1 represents high-level analysis of key features offered in social VR applications that we accumulated from the expert interviews. Based on this analysis, we made further claims about what kinds of embodied experiences these platforms support and what impact they have on social interactions in multi-user virtual environments. Along with a range of general commonalities across features such as embodied locomotion, custom world creation affordances, personal space mechanics, avatar representations and social mechanics, there are many nuances in design approaches to each subcategory of those features. The latter, in turn, make all of these social VR application different from one another.

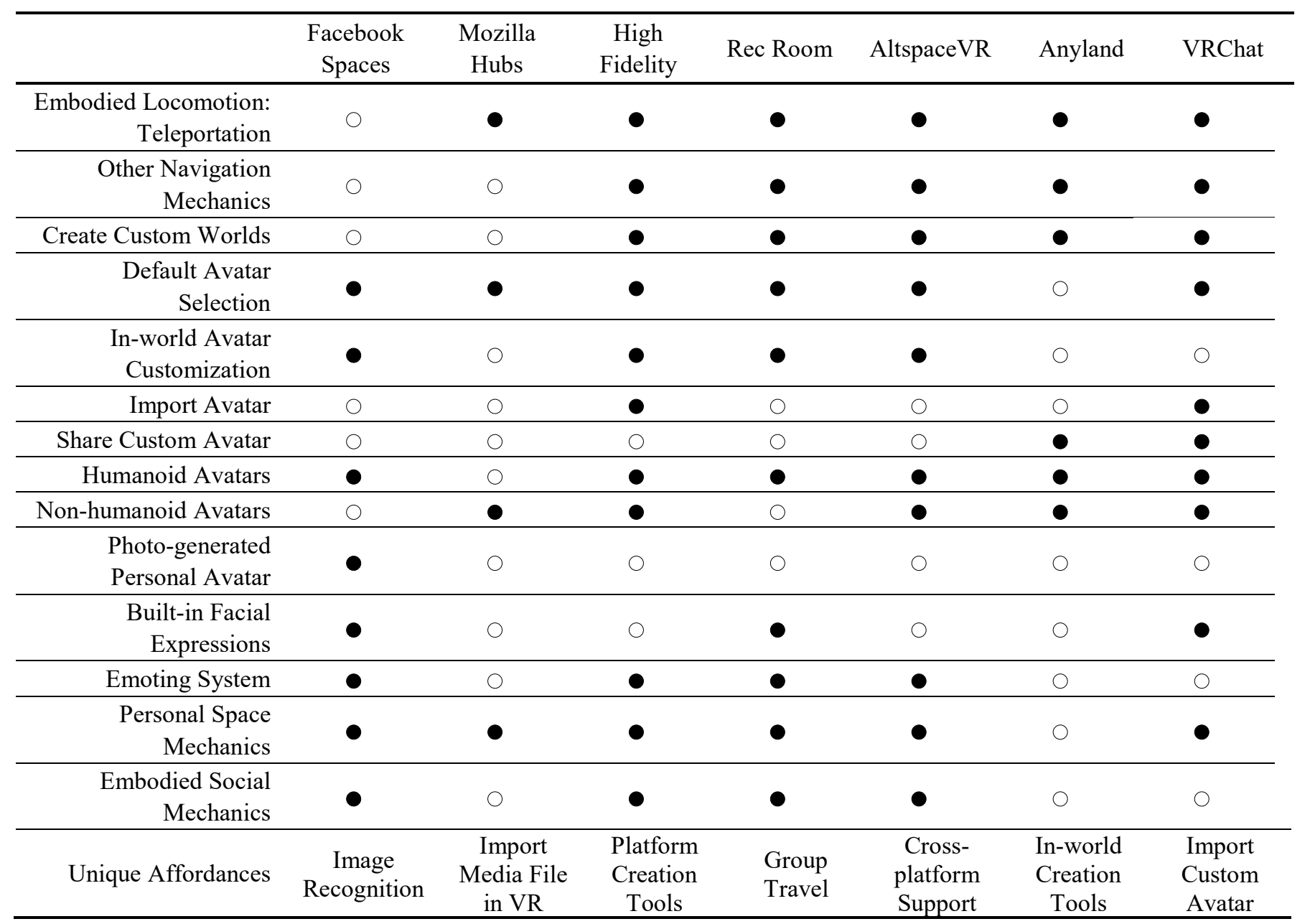

Table 1: High Level Analysis of Avatar Affordances in Social VR Application. 


\section{Embodied Locomotion}

One of the first things a user learns about their avatar is how to control them in the corporeal-virtual navigation mechanics within social VR worlds. Teleportation was one of the dominant forms of avatar locomotion utilized in all six social VR applications. Using hand held controllers, users can move their avatars by pointing to where they want to move, transitioning towards that destination in real-time. The most popular design practice for teleportation that we observed was teleportation with a parabolic pointer. Parabolic teleportation allows users to teleport on top of objects that are elevated in relation to avatar's position in space. AltspaceVR utilized an alternative approach to the parabolic pointer, in which users teleport using a line pointer [Figure 3].

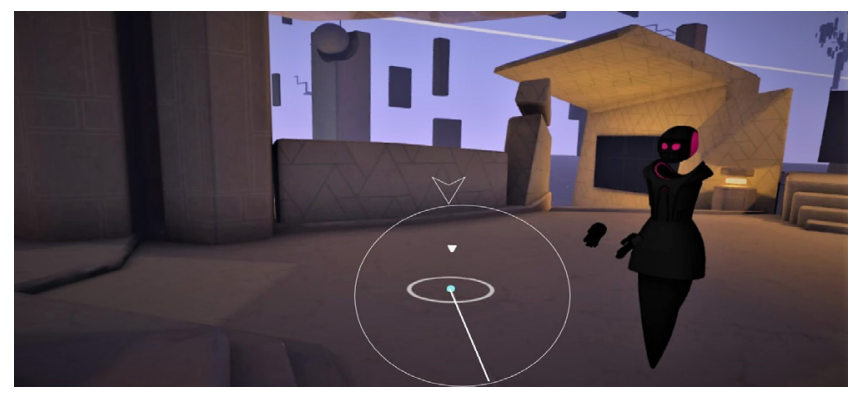

Figure 3: Teleportation Mechanic Demo in AltspaceVR.

We found that teleportation was closely related to social mechanics of avatars, such as blocking. Many experts noted that the distance for teleporting should be carefully considered in design practice for social VR. In most cases, users could teleport within their proximity zone. It was an intentional design decision to resolve some moderation issues. Most social VR applications, such as Rec Room, AltspaceVR, and High Fidelity have in-world moderators who ensure the comfort and safety of their users within public virtual spaces. Moderators have a right to block or ban a user if one breaks the application's code of conduct. For moderators to block a user, they need to stay within their proximity and point a context laser at them for a pop-up menu to appear. As communities became larger and more experienced, users learned to avoid moderators by 'running away' from them using the teleporting mechanic. For instance, a Rec Room moderator would have to 'chase' a user across the VR space to block or report them, until designers decided to constrain the distance for the teleporting mechanic.

Three social VR designers found that teleportation for far distances negatively affected social interaction with users who shared the same virtual environments. In Rec Room users have an option to adjust the distance of their avatar's parabolic beam, which might be useful for instances when they travel into other environments of different size and scale. To do that, users need to pull up a setting menu on their watch and set the teleport bubble to 'small', 'medium', or 'large'.
The third person walking mechanic was one of the unique locomotion affordances supported exclusively in VRChat. As one expert noted, this mechanic could be a solution to minimize motion sickness, as opposed to first-person locomotion in VR. Content creators for VRChat might find this mechanic helpful in situations when they need to test out the performance of their self-created avatars [Figure 4].

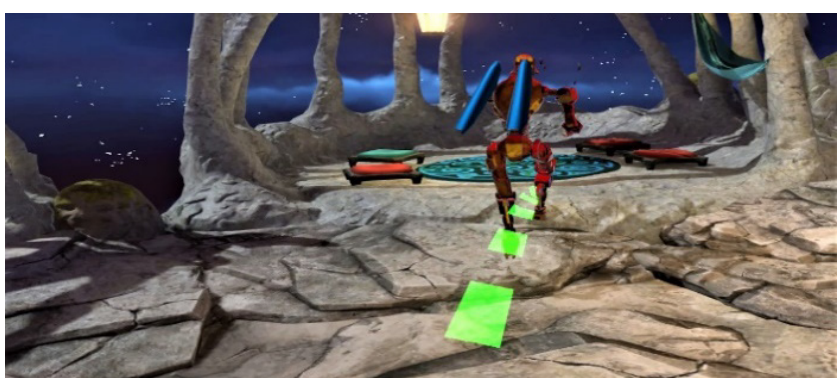

Figure 4: Third Person Walking Mechanic in VRChat.

\section{Avatar Aesthetics}

Researchers held a lot of discussions on how realistic avatars needed to appear for users to effectively perform in social contexts within virtual spaces [6],[11],[20],[39]. The design choices for avatars' visual fidelity were primarily driven by performance constraints. In particular, creators at Mozilla Hubs and AltspaceVR, emphasized that the aesthetics and the art style of avatars needed to support device interoperability. The appearance of avatars in those applications was fairly simplistic - designers intentionally scaled down the polygons and geometry of 3D objects to ensure users did not run into any performance issues across platforms.

Some designers viewed the appearance of users' avatars as a protective shield from harassment in virtual spaces, especially for women. To ensure user safety, most creators wanted to avoid hyper-sexualized appearances in avatar choices. As a Senior Product Manager at AltspaceVR noted, their initial design approach to avatars was to "make them look more neutral and approachable for everyone else within the virtual space".

In contrast to other social VR applications, avatar appearances in VRChat were highly valuable to their virtual community. Most users in VRChat could create and upload their own avatars into VR spaces. Users in VRChat have more freedom to experiment with different shapes, sizes, art styles, scale, and even behaviors for their avatars. One example that particularly stood out was the practice of designing puppet avatars. Puppet avatars, as opposed to traditional avatars in social VR, are controlled by holding a smaller version of an avatar body (puppet) in one hand using a VR controller. According to Ventrella, 'puppeteering' is described as the act of controlling an inanimate object or a virtual character in real-time, to create the illusion of life [38]. As an Avatar Designer in VRChat said, puppet avatars could help new social VR users overcome social anxiety and serve as one of the social lubricants in virtual 
spaces. In social VR, the non-verbal behavior of avatar puppets reflects the behavior of a user in real time [Figure 5].

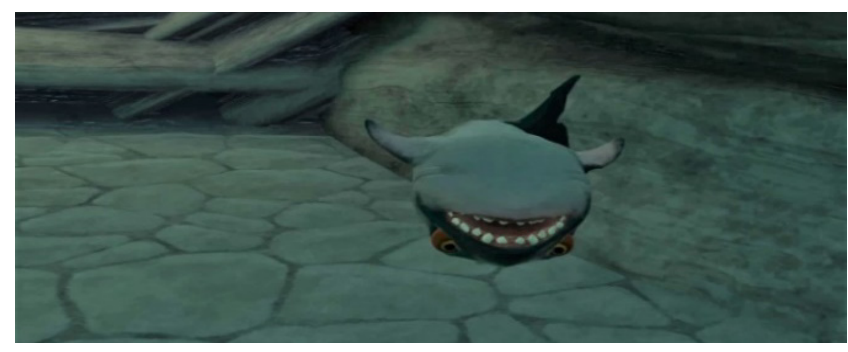

Figure 5: Puppet Avatar Demo in VRChat.

The avatar system designed in Facebook Spaces was one that was on the other end of the spectrum in regard to its relation to realism [Figure 1].

\begin{abstract}
... "To achieve the feeling of being present with your friends in VR, you need to be able to bring some elements of your real-world identity. Attaching body and arms to head and hands makes your avatar feel more realistic and give it presence and volume in the space." [Christophe Tauziet, Former Lead Designer of Facebook Spaces]
\end{abstract}

\section{Personal Space}

Avatars can be viewed as a 'protective shield' within multiuser virtual spaces [4]. In social VR the mechanics for personal space function as an invisible 'bubble' that avatars wear. In Facebook Spaces it's called a 'personal safety bubble'.

... "We created a personal safety bubble that acts as a shield and protects you from anything or anyone entering it." [Christophe Tauziet, Former Lead Designer of Facebook Spaces]

Similarly, Rec Room users have an option to turn on the socalled ignore bubble around their avatars that outlines the borders of avatar's personal space. Users can adjust the size of the ignore bubble based on their own preferences and personal level of comfort. If other users appear within the proximity of that ignore bubble, their avatar will turn into a ghost-looking shape that disappears as they move closer towards each other [Figure 6].

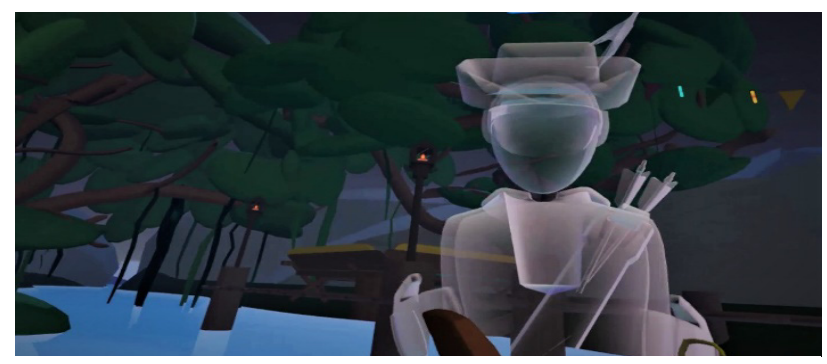

Figure 6: Personal Space Mechanic in Rec Room.

Personal space bubbles and social mechanics such as 'blocking' appeared in strong relation to each other. A common design intent was to provide users with a means to protect their personal space. The specifics of blocking mechanics varied across applications. In Mozilla Hubs for example, the 'pause' mechanic performs the function of blocking someone in the space. It 'freezes' the state of the room in Hubs and gives users the freedom to move in and out of the experience.

Another variation of blocking mechanic is represented by 'muting' and 'reporting' a user in AltspaceVR. To mute or report a user, a person could either use a line pointer to hover over a person's avatar name tag or pull up the main menu. An alternative option to the ignore bubble mechanic was a 'stop gesture' in Rec Room. The stop gesture could be performed by raising one of the hand-held VR controllers in VR space. For instance, if a user appears too close to another person's avatar, they could use a stop gesture as a prompt response to inform them about their personal space limits. This stop gesture mechanic appeared more quick and intuitive as opposed to accessing the ignore bubble and muting mechanics. Most designers found personal space and blocking mechanics essential to the initial user experience in social VR.

A unique approach to the design of personal space was introduced in Anyland. Instead of having 'bubbles' as in other social VR applications, Anyland users utilize their inworld private home spaces as a personal bubble. If a user is a creator of an 'area', where they host other users, then they have an ability to lock any disturbing user out of their area. To do this, they need to click on a door icon in their menu. Anyland completed many iterations around the design of this feature. They intentionally decided not to implement in-world personal space bubbles because of the intricate aspects of the community dynamics that that feature may potentially cause.

\begin{abstract}
... "as soon as you offer a defense tool, people will, or a portion of people will become curious about how to circumvent that. And then they will invent all kinds of weapons. And I don't know, whatever they can try to use to pierce that personal defense bubble... And then it becomes the problem of a person who wants to defend themselves, which is the exact reverse of what we want." [Philipp Lenssen, Co-founder and Developer of Anyland]
\end{abstract}

\section{Social Mechanics}

In High Fidelity a handshake with another person's avatar results in a friendly explosion of pixels, which means the user became friends with that person. In Rec Room a similar functionality is triggered by a fist bump in VR space, except this action serves as a VIP ticket to a private party in another room. In most social VR applications, including High Fidelity, Anyland, and VRChat, the mechanics for traveling into different virtual environments are performed through the menu in VR. The challenge for most creators, however, was to make this experience more embodied and socially engaging. AltspaceVR introduced a party portal mechanic as a unique solution to this design challenge. A party portal in AltspaceVR can also be viewed as part of the embodied navigation modalities cluster that we discussed earlier. To travel into virtual environments together with a party of 
users, most applications require them to become friends first, allowing them to send an invite to a virtual location they want to go. Party portal allows users to travel together to other environments with a group of people who do not necessarily need to be friends with each other [Figure 7].

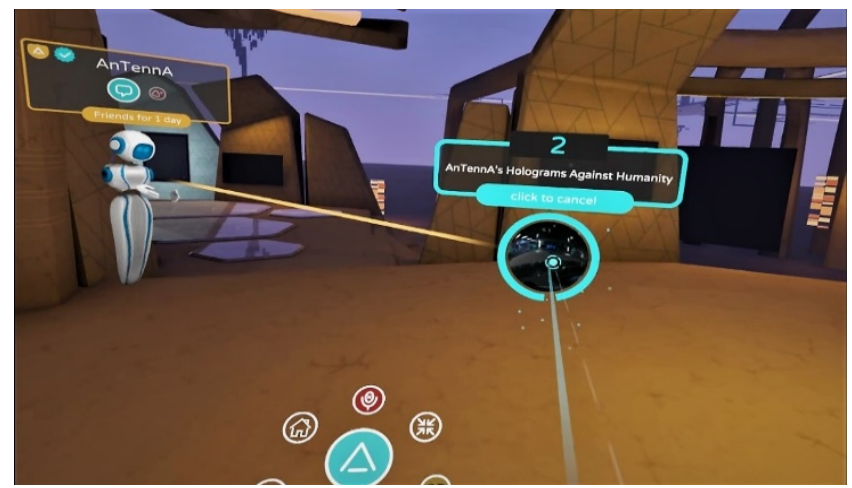

Figure 7: Party Portal Demo in AltspaceVR.

In Facebook Spaces, AltspaceVR, High Fidelity and Rec Room, users can communicate with each other through their avatars' emoting system. In Rec Room, for instance, if a user holds down a menu button on their hand-held controller, an 'Expresso' menu will pop up. They can then select a nonverbal expression by moving their hand towards one of the displayed emoji images. When selected, their avatar would make the selected facial expression. Such non-verbal behavior is accompanied by a cloud bubble feature that appears above their avatar to display an image of the selected emoji [Figure 8].

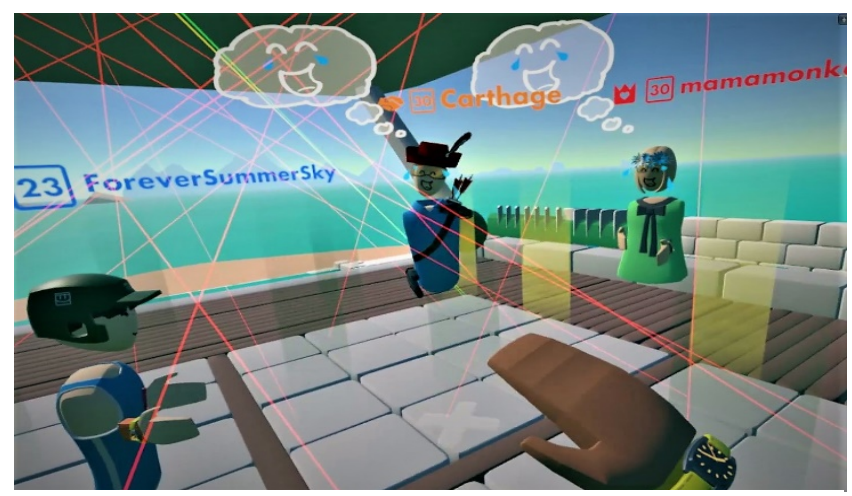

Figure 8: Avatars' Non-verbal Behavior or the 'Expresso' System in Rec Room.

The options for the avatar's non-verbal expressions in Rec Room change depending on the kinds of social contexts or environments users are in. If a user completed a quest as a party member, the options for expressions would change from the regular ones ('sad', 'smile', 'love', etc.) to 'forward', 'retreat', 'enemy', 'health', and 'watch out'. In addition, as one designer noted, some objects in the environment could trigger specific kinds of avatar expressions. For instance, if someone threw a camera into one's avatar's face, the "I just got hit in the face!" expression would appear. The same expression would appear if someone accidently smacked a user's avatar. A spatial audio feature in Rec Room compliments the avatar emoting system in a small group setting. In a large group setting with up to 6 or 10 people standing next to each other, it is sometimes challenging to identify who is speaking. In this type of social context, it is often hard for a user to notice an avatar's lips animations. To address this challenge, designers integrated the speech lines feature. It would appear above users' avatars to help them quickly identify who is currently speaking and who is not. Similarly to Rec Room, AltspaceVR creators looked into the specifics pertinent to various types of communication contexts when crafting avatar non-verbal behavior in VR.

\begin{abstract}
... "you can think of communication as being instructional, when someone is up on the stage and doing a presentation. You can think of communication as being conversational, where we're trying to give each other gestures or nods, or some signs of understanding. You can think of it as being productive and collaborative communication, where you're trying to be more centered on a task, or an activity. You can think of it as being more intimate, like giving someone a hug, or maybe look in their eyes, which is more nuanced type of communication. Those are the various categories of communication that we consider in general when we think about avatar design. There's different fidelities of social VR and VR that can support some of those better, than the others can today." [Ishita Kapur, a Senior Product Manager at AltspaceVR]
\end{abstract}

We found an interesting feature in regard to an avatar's expressivity towards others in social contexts in VRChat. Along with handshake and high five social mechanics, users often pat someone's avatar head as a gesture of appreciation or a hug. That type of behavior primarily originated internally from the VRChat community. While it is not something that people normally do in real life, in VRChat environments it has become a social norm. As one interview expert argued, to give a hug or be hugged by someone who is not your friend in VRChat could seem strange and sometimes discomforting to your personal space. Instead, patting an avatar's head appeared much easier and less socially awkward.

Overall, we found that social mechanics often involve personal space mechanics. The primary examples of those were blocking, muting, flagging and reporting. Most of these mechanics were integrated as part of VR menus, which made the experience less embodied in comparison to non-verbal behavioral systems, such as emoting and hand gestures. All applications offered a variety of tools for their users to 'protect' their VR experience from unwanted interactions, in the form of reporting and blocking someone. In most social VR applications, to block or report someone, users need to point a context laser at them in VR and select a 'block/mute' option from the menu. Alternatively, users can call a tab from the main menu that would display a list of concurrent users who share or shared the same VR space and then block or report them from there. 


\section{An Avatar's Relationship to Virtual Identity}

An avatar's aesthetics, appearance, customization and representation aspects strongly correlate with a user's virtual identity and the perceptions of others. As most experts agree, the way avatars act and look, and the achievement attributes they decide to display make a large contribution to one's identity.

\begin{abstract}
... "I've heard stories about conversations my female friends have had with teams that are dominated by white/Asian males who work on apps with low customization of largely white/thin avatars. This causes me to be unsure if men understand and identify with the importance of visual representation in a social setting as much as women do. I'm part of that woman group, and we've been trained by society our whole lives that our visuals define us. We're bombarded by media to alter our outer appearance in order to define ourselves. I need to make myself stand out. This is (one reason) why I place so much importance on visual representation of avatars. With this said, it's important to me to help people craft their own identities. I've made myself into a storm cloud in High Fidelity." [Alexia Mandeville, UX Designer]
\end{abstract}

Experts who were affiliated with cross-platform social VR applications (Mozilla Hubs, AltspaceVR, High Fidelity) identified the following design challenges in fostering users' identity with their avatars: "How do we ensure that people have the freedom that they should have to take on the form they want?"; "Along with providing a wide range of avatar customizability options, how would we allow people to bring in their own avatars without bogging the platform down?"; "How do we ensure that users feel comfortable with the arrived tool set for 3D modeling and rigging?".

Mozilla Hubs creators also believed that most people that created their own avatar, treated it as their own personal brand, as their identity. As in real life situations (professional and non-professional environments), in VR users might want to have multiple identities for different social contexts. Experts at Mozilla Hubs strongly disagreed with a view on multiple identities as a sign of a lack of integrity. To them, it was quite the opposite-- they believed that people who want to explore different identities and try on different personas are acting in a natural way. Greg Fodor, a Software Engineer at Mozilla Hubs added: “... it's really important to us that we don't box people in from having to be forced in a certain mindset in regard to how their avatar expresses their identity."

Designers of Facebook Spaces aimed to bridge to real-world identity by utilizing image recognition software. They believed that avatar styles along with customization options were "... quite flattering and tend to be more of an aspirational version of who you are" [Christophe Tauziet, Former Lead Designer of Facebook Spaces].

Alternatively, Anyland users do not embody any avatar, but an empty skeleton at the beginning of their experience. Philipp Lenssen, a Co-Founder and Developer of Anyland, argued that creating your own avatar is about 'inventing your identity'. Their creators believed that there is always a chance users would not relate to any given default set of avatars. Instead, they wanted to encourage users to explore themselves and think about who they really wanted to be in VR. By providing in-world tool sets for avatar creation, Anyland creators believed that it was a much more interesting, creative, and entertaining process for their users to think about their own selves in VR. In VRChat and High Fidelity users could upload their own avatars created using third-party applications. Alternatively, in-world building tools in Anyland allowed users to not only create their own avatar models, rig and animate them, but also create virtual spaces without taking their VR headsets off. As a shortcut to the creative process of creating an avatar in Anyland, their community created a body shop space that all users could go visit, display, and/or share their hand-made avatars, along with customization items for others to try on.

Another interesting observation that experts registered among social VR communities was the impact of avatar's appearance and behavior on users' perception of others. For example, users in Rec Room could earn rare items if they completed certain quests and then put those items on their avatars to wear. As Rec Room creators agreed, it was a way for users to communicate their identification with a certain game/quest community. Similarly, High Fidelity users whose avatars wore different outfits or users who represented themselves in a certain way might often become a conversation starter in social VR communities.

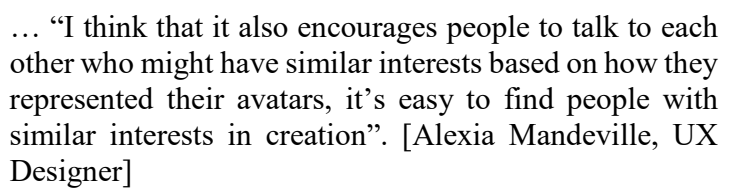
represented their avatars, it's easy to find people with similar interests in creation". [Alexia Mandeville, UX Designer]

\section{DISCUSSION}

The results accumulated from the expert interviews supported most of the insights we drew from the existing research on avatars, with which we propose to consider the design of avatars in the commercial social VR medium from the systemic stand point. We believe that avatars in social VR contexts represent a system with a variety of multivalent and interrelated dimensions that impact the formation of embodied experiences in multi-user virtual environments. Those factors included (1) embodied locomotion, (2) avatar aesthetics, (3) personal space mechanics, (4) social mechanics, and (5) an avatar's relation to virtual identity. Most of those factors were often found interlinked in fairly sophisticated ways, pertinent to the unique constraints and affordances of each social VR platform. The analysis of systemic dimensions in avatar design resulted in a list of high-level design consideration areas. It is worth noting that this list is not exhaustive and is based on the emerging design practices of social VR creators who participated in our research. 


\section{Key Design Topics and Opportunities}

1. Avatars in social VR exist in a system of personalization alternatives and/or customization options, in which selection from the constrained set of customizable options could only be understood from within a particular slice of social VR ecology.

2. Various mechanics of avatars systemically interact with other features in social VR.

3. Avatar affordances play a systemic role in communication and identity signaling.

While these topics may seem similar to those discussed previously concerning non-VR avatars, differences in avatar design can have a significant impact on resulting embodied user experience in social VR, which we would like to elaborate here.

The design approach to an avatar system in social VR depends on the high-level goal and priorities of the creators. In AltspaceVR, for example, the avatar system was designed to support the cross-platform compatibility feature. To meet the performance requirements of that feature, users were given a default set of low-poly avatar shapes to choose from with some variation for customization. Whereas in Anyland, first time users could often be identified by wearing an empty skeleton with no avatar body. This type of experience was designed to support this platform's unique affordance utilization of in-world creation tools.

Various mechanics of avatars systemically interact with other features of embodied experiences in social VR. For instance, the design of an avatar's locomotion mechanic can affect the usability of the blocking mechanic. If the distance for teleportation was designed without regard to the scale and spatial architecture of the virtual environment, an alternative approach to the blocking mechanic would be recommended to integrate for large and open spaces in VR. Rather than 'chasing' a user within that environment to point a context laser at them, social VR creators and researchers might want to consider other approaches to implement. It could be a feature that sets a limit for teleportation distances, and/or some sort of an environmental cue that communicates to first time users on what actions to take to protect their VR experience from unwanted social interactions.

The design of avatar affordances can also have a significant impact on communication and identity signaling. For example, the option to wear a puppet avatar in VRChat was found to help reduce social anxiety, in particular for firsttime users. This type of emerging design practice is underexplored in scholarly work on puppet avatars in multi-user virtual environments. Furthermore, the design of a rare item/outfit that an avatar could wear in a social VR environment not only reinforced user's identification with their avatar, but also fostered social interactions within the virtual community. It allowed users to shape a desired perception by others by having their avatar wear that item.
Thus we believe that the proposed framework for studying avatars as a system of embodied experiences provides the research community and design practitioners with a means to characterize the social VR space, and allows us to think about the affordances of a specific social VR platform, its input mechanisms (e.g. VR vs. keyboard/touchpad), as well as the design of the in-world qualities of avatars.

\section{CONCLUSION}

In this paper, we presented a set of emerging design practices for avatar systems in the commercial social VR ecology, based on insights accumulated from in-world interviews with creators of popular social VR applications. The research outcomes are aimed at better understanding the challenges, constraints, and trade-offs among the existing avatar design practices.

We proposed a new approach to studying avatars as a systemic construct of multivalent relationships between various factors that shape embodied experiences and social interactions in social VR. Though this approach is preliminary, it introduces new avenues for empirical user research. For example, interviews with users could further contextualize the design considerations of avatar systems in social VR, and validate design practices that support or constrain embodied experiences in social contexts.

As Nardi has noted, the affordances that designers and other practitioners deem important will inevitably shape an extensive portion of human social interactions today and in the future [27]. Taylor's question about "What will we look like and who will be in a world where technology so deeply intersects our lives?" remains open [38]. In the long term, future user research on the persistence of avatar appearance in different social contexts across different social VR platforms may identify new dimensions of embodied experience, and enrich the proposed concept of avatar systems. What kind of persistence do users need to recognize each other over repeated encounters? What degree of diversity in avatar systems is needed for users to distinguish each other in small and large VR communities? [30] As Nate Mitchell, Oculus co-founder and Product VP once noted, "Recognizing your friend's avatar in social VR for the first time is a magical experience" [41].

\section{ACKNOWLEDGMENTS}

We thank all the experts for their knowledgeable contribution in this research, and our Social Emotional Technology Lab team at University of California Santa Cruz. This research was supported by a grant from Mozilla. We extend special thanks to Lars Bergstrom, Blair MacIntyre, Miriam Avery, Jofish Kaye, Greg Fodor, Jim Conrad, and the rest of the Social Mixed Reality team at Mozilla. We also thank our anonymous reviewers for their feedback and contributions. 


\section{REFERENCES}

[1] Bailenson, J.N. et al. 2004. Avatars. Berkshire encyclopedia of human-computer interaction, 64-68.

[2] Bailenson, J.N. et al. 2006. The Effect of Behavioral Realism and Form Realism of Real-Time Avatar Faces on Verbal Disclosure, Nonverbal Disclosure, Emotion Recognition, and Copresence in Dyadic Interaction. Presence: Teleoperators and Virtual Environments 15, 4: 359-372. https://doi.org/10.1162/pres.15.4.359

[3] Birk, M. V et al. 2016. Fostering Intrinsic Motivation through Avatar Identification in Digital Games. CHI: 2982-2995. https://doi.org/10.1145/2858036.2858062

[4] Bosch-Sijtsema, P.M. et al. 2013. Professional Virtual Worlds Supporting Computer-Mediated Communication, Collaboration, and Learning in Geographically Distributed Contexts. IEEE Transactions on Professional Communication 56, 2: 160-175. https://doi.org/10.1109/TPC.2012.2237256

[5] Clarke, C.P. 2012. Second Life in the library: an empirical study of new users' experiences. Program 46, 2: 242-257. https://doi.org/10.1108/00330331211221864

[6] Davis, A. et al. 2009. Avatars, People, and Virtual Worlds: Foundations for Research in Metaverses. Association for Information Systems. Retrieved September 19, 2018 from https://aisel.aisnet.org/jais/vol10/iss2/1

[7] Ducheneaut, N. et al. 2009. Body and mind. In Proceedings of the 27th international conference on Human factors in computing systems - CHI 09, 1151. https://doi.org/10.1145/1518701.1518877

[8] Economou, D. et al. 2017. User experience evaluation of human representation in collaborative virtual environments. Pers Ubiquit Comput 21: 989-1001. https://doi.org/10.1007/s00779-017-1075-4

[9] Felnhofer, A. et al. 2014. Physical and social presence in collaborative virtual environments: Exploring age and gender differences with respect to empathy. Computers in Human Behavior 31: 272-279. https://doi.org/10.1016/j.chb.2013.10.045

[10] Fox, J. et al. 2009. Virtual Experiences, Physical Behaviors: The Effect of Presence on Imitation of an Eating Avatar. Presence 18, 4: 294-303. Retrieved April 3, 2018 from https://www-mitpressjournalsorg.oca.ucsc.edu/doi/pdf/10.1162/pres.18.4.294

[11] Garau, M. 2003. The Impact of Avatar Fidelity on Social Interaction in Virtual Environments. University College London. Retrieved April 3, 2018 from http://citeseerx.ist.psu.edu/viewdoc/download?doi=10 1.1.459.8694\&rep=rep1\&type $=$ pdf
[12] Garau, M. et al. 2005. The Responses of People to Virtual Humans in an Immersive Virtual Environment. Presence 14, 1: 104-116. Retrieved April 3, 2018 from https://www-mitpressjournalsorg.oca.ucsc.edu/doi/pdf/10.1162/1054746053890242

[13] Gonzalez-Franco, M. et al. 2015. Framework for remote collaborative interaction in virtual environments based on proximity. 2015 IEEE Symposium on 3D User Interfaces, 3DUI 2015 Proceedings, June 2015: 153-154. https://doi.org/10.1109/3DUI.2015.7131746

[14] Gonzalez-Franco, M. et al. 2016. The Neurological Traces of Look-Alike Avatars. Frontiers in Human Neuroscience 10. https://doi.org/10.3389/fnhum.2016.00392

[15] Heidicker, Paul; Langbehn, E. 2017. Influence of Avatar Appearance on Presence in Social VR. Proceedings of IEEE Symposium on $3 D$ User Interfaces (3DUI), University of Hamburg: 1-3. Retrieved from https://basilic.informatik.unihamburg.de/Publications/2017/HLS17/template.pdf

[16] Isbister, K. 2006. Better Game Characters by Design: A Psychological Approach. Elsevier/Morgan Kaufmann Series in Interactive 3D Technology. https://doi.org/10.1016/B978-1-55860-921-1.50012-0

[17] Isbister, K. et al. 2010. Designing games for learning. In Proceedings of the 28th international conference on Human factors in computing systems - CHI '10, 2041. https://doi.org/10.1145/1753326.1753637

[18] Isbister, K. et al. 2015. Guidelines for the Design of Movement-Based Games and Their Relevance to HCI. Human-Computer Interaction 30, 3-4: 366399. https://doi.org/10.1080/07370024.2014.996647

[19] Kafai, Y.B. et al. 2010. Your Second Selves. Games and Culture 5, 1: 23-42. https://doi.org/10.1177/1555412009351260

[20] Latoschik, M.E. et al. 2017. The Effect of Avatar Realism in Immersive Social Virtual Realities. 17, 10. https://doi.org/10.1145/3139131.3139156

[21] McVeigh-Schultz, J. et al. 2018. What's It Mean to "Be Social" in VR?: Mapping the Social VR Design Ecology. In Proceedings of the 2018 ACM Conference Companion Publication on Designing Interactive Systems (DIS '18 Companion): 289-294. https://doi.org/10.1145/3197391.3205451

[22] McVeigh-Schultz, J. et al. 2019. Shaping Pro-Social Interaction in VR: An Emerging Design Framework. In 2019 CHI Conference on Human Factors in Computing Systems Proceedings (CHI 2019). https://doi.org/10.1145/3290605.3300794

[23] Michael, N. et al. 2014. Model-Based Generation of Realistic 3D Full Body Avatars from Uncalibrated Multi-view Photographs. 11. Retrieved April 3, 2018 
from https://rd-springer-

com.oca.ucsc.edu/content/pdf/10.1007\%2F978-3662-44654-6_35.pdf

[24] Morie, J.F. 2014. Avatar appearance as prima facie non-verbal communication. In Nonverbal Communication in Virtual Worlds, Joshua Tanenbaum, Magy Seif El-Nasr, and Michael Nixon (Eds.). Nonverbal Communication in Virtual Worlds: 77-102. Retrieved September 19, 2018 from https://dl.acm.org/citation.cfm?id=2812755

[25] Perry, T.S. 2016. Virtual reality goes social. IEEE Spectrum 53, 1: 56-57. https://doi.org/10.1109/MSPEC.2016.7367470

[26] Rajan, V. et al. 2001. A Realistic Video Avatar System for Networked Virtual Environments. Retrieved August 30, 2018 from https:/www.semanticscholar.org/paper/A-RealisticVideo-Avatar-System-for-Networked-RajanSubramanian/7871850926b1426365fb0d39a7f5bbc7a dd7f290

[27] Redfern, S. et al. 2013. Collaborative Virtual Environments to Support Communication and Community in Internet-Based Distance Education. Retrieved September 3, 2018 from https://aran.library.nuigalway.ie/handle/10379/4076

[28] Roth, D. et al. 2017. Avatar Realism and Social Interaction Quality in Virtual Reality. VRST'17 Proceedings of the 23rd ACM Symposium on Virtual Reality Software and Technology Article No: 2. Retrieved April 3, 2018 from http://www.hci.uniwuerzburg.de/download/2016-ieee-vr-posterinteraction-qualilty.pdf

[29] Saldana, J. 2016. The Coding Manual for Qualitative Researchers.

[30] Schroeder, R. 2002. The social life of avatars : presence and interaction in shared virtual environments. Springer. Retrieved April 3, 2018 from https://books-google-com.oca.ucsc.edu/books?id=d7vegnIgJsC\&lr $=\&$ source $=$ gbs_navlinks_s

[31] Schroeder, R. et al. 2006. The Usability of Collaborative Virtual Environments and Methods for the Analysis of Interaction. Presence: Teleoperators and Virtual Environments 15, 6: 655-667. https://doi.org/10.1162/pres.15.6.655

[32] Schroeder, R. 2010. Being There Together. Oxford University Press. https://doi.org/10.1093/acprof:oso/9780195371284.00 1.0001

[33] Sivan, Y. et al. 2014. Fostering Team Creativity in Virtual Worlds. Journal of Virtual Worlds Research 7, 32. https://doi.org/10.4101/jvwr.v7i3.7062

[34] Slater, M. et al. 2000. Small Group Behaviour in a Virtual and Real Environment: A Comparative Study.
University College London: 31. Retrieved April 3, 2018 from http://www-

dept.cs.ucl.ac.uk/staff/m.slater/Papers/inhabit-presrev.pdf

[35] Stephenson, N. Snow crash. Retrieved September 3, 2018 from https:/www.amazon.com/Snow-CrashNovel-Neal-Stephenson-ebook/dp/B000FBJCJE

[36] Tanenbaum, J. et al. 2014. Nonverbal communication in virtual worlds : understanding and designing expressive characters. ETC Press. Retrieved September 12, 2018 from https://dl.acm.org/citation.cfm?id=2812773

[37] Trepte, S. et al. 2010. Avatar Creation and Video Game Enjoyment. Journal of Media Psychology 22, 4: 171-184. https://doi.org/10.1027/1864$1105 / \mathrm{a} 000022$

[38] Ventrella, J. 2014. Virtual gaze: the communicative energy between avatar faces. In Nonverbal Communication in Virtual Worlds, Joshua Tanenbaum, Magy Seif El-Nasr, and Michael Nixon. Nonverbal Communication in Virtual Worlds: 61-75. Retrieved September 19, 2018 from https://dl.acm.org/citation.cfm?id=2812754

[39] Vinayagamoorthy, V. et al. 2004. An eye gaze model for dyadic interaction in an immersive virtual environment: Practice and experience. Computer Graphics Forum 23, 1: 1-11. https://doi.org/10.1111/j.1467-8659.2004.00001.x

[40] Waltemate, T. et al. 2018. The Impact of Avatar Personalization and Immersion on Virtual Body Ownership, Presence, and Emotional Response. IEEE Transactions on Visualization and Computer Graphics 24, 4: 1643-1652. https://doi.org/10.1109/TVCG.2018.2794629

[41] Webster, A. 2016. Oculus Avatars look like the future of video game character creation - The Verge. The Verge. Retrieved September 15, 2018 from https://www.theverge.com/2016/10/6/13177082/oculu s-rift-avatars-vr-virtual-reality 